\title{
Gut Microbiome Derived Metabolites to Regulate Energy Homeostasis: How Microbiome Talks to Host
}

\author{
Hariom Yadav ${ }^{1 *}$, Shalini Jain ${ }^{1}$, Laura Bissi ${ }^{2}$ and Francesco Marotta ${ }^{3}$ \\ ${ }^{1}$ National Institute of Diabetes and Digestive and Kidney Diseases, National Institutes of Health, Bethesda, Maryland, USA \\ ${ }^{2}$ Milano Medical Gender Healthy Aging Unit by Genomics, Milano, Italy \\ ${ }^{3}$ ReGenera Research Group for Aging Intervention and Milano Medical, Milano, Italy
}

Gut microbiome recently known to be one the major player in regulating several physiological functions in normal and pathological conditions of human diseases [1]. There is always a curios question remains to non-expert in the field that how gut microbiome interacts with host cells and impacts cellular metabolism. For experts, unraveling these facts and discussing them, always brings new viewpoints in the field to further study that can be critical to uncover new drug targets and biomarkers of metabolic diseases. The prevalence of two metabolic diseases i.e., obesity and type 2 diabetes are on peak, and are associated with several life threatening consequences like cardiovascular diseases, brain disorders and cancer [2]. Gut microbiome contributes significantly in the pathology of obesity and diabetes $[3,4]$. As initial studies showed that transplantation of obese gut microbiome to distinct recipient have ability to transfer obesity from donor to recipient in mice, suggesting gut microbiome carries certain intrinsic factors that interacts with host metabolism and develops metabolic derangements. Several pathways have been proposed to be modulated by gut microbiome to cause metabolic syndrome, some of them are as follows; 1) gut microbiome helps to harvest extra energy from food that further increase extra energy accumulation in fat tissues, 2) gut microbiome mediated endotoxemia to create low grade inflammation and 3) production of certain detrimental metabolites that implicates dysregulation of several cellular function to aggravate disease progression $[3,5]$. For example, gut microbiome-derived TMAO induces cardiovascular diseases, acetate impairs insulin secretion and LPS escalates obesity and type 2 diabetes via increasing low grade inflammation [5-7]. To answer the question how microbiome interacts with host cells and impact metabolic function, later point explains the better link between gut microbiome and host in normal physiology.

Considering the fact that, there is a very viscous, hydrophobic and thick mucus layer into gut linings throughout the gastrointestinal tract that makes a germ free zone between microbiome and host cells. Hence, gut microbial bodies have very little chances to interact with host cells. But metabolites that are produced into gut via active fermentation can diffuse easily from mucus layer and can interact with host cells. This interaction might occur in one of these two mechanisms or both i.e., 1) either metabolites bind with host cell receptors to activate or suppress respective receptor mediated signaling pathways or 2) metabolites can be absorbed into cells and can enter into intracellular metabolic flux to change metabolic function of cells. These metabolite-mediated common pathways are vital in communication between host cells and microbiome, to control an array of metabolic functions. Gut microbiome produces large number of metabolites that interacts with cells of mucosal immune system, enteric nervous system and enteroendocrine cells [8]. In addition these metabolites can also enter into circulation and reach to different metabolic organs i.e., brain, liver, pancreas, adipose tissues and skeletal muscles (Figure 1), and participate changes in metabolic functions. Microbiome character as beneficial and pathological depends on ability of microbes to produce types of metabolites, as some are beneficial while others can be detrimental. For example, certain metabolites like TMAO, acetate and
LPS are pathological, while other metabolites like butyrate, propionate and several amino acid derivatives are known for beneficial effects.

Now gut is also known as a second brain due to the facts that gut microbiome and enteric nervous system produce an array of neurotransmitters, i.e., $95 \%$ serotonin and 50\% dopamine in our body comes from gut. Gut derived neurotransmitters not only modulate local enteric nervous system but also controls major functions in Central Nervous System (CNS). It is also interesting to note that gut microbiome produces several other neurotransmitters like GABA, 5'-hydrotyrosine, acetylcholine, tryptophan that modulate energy homeostatic centers in brain to control whole body metabolic functions via modulating energy/food intake and energy expenditure. Gut microbiome field is progressing very fast to unfold interactions between host and microbiome to regulate energy homeostatic points, but substantial efforts are needed to establish the therapeutical and diagnostic importance of gut microbiome and its metabolites for obesity and diabetes. In our views, some of the areas are of urgent need to studied for better understating the biology i.e., 1) defining interaction of gut microbiome with host cells via receptor or intracellular signaling, 2) establish a network of enteric nervous system to connect with other metabolic organs i.e., adipose tissues, liver, pancreas, muscles to uncover their local communication using neuronal physical threads, 3) design precise antibiotics to kill detrimental microbes for desired microbiome modulation, and 4) develop new probiotics and prebiotics to restore depleted beneficial microbial population into diseased gut.

These few intriguing points represent huge area of research in this direction, and much remains to be learned regarding contribution of gut microbiome, diet and antibiotics use to offer importance in modulation of host metabolism. Further studies will explore these directions and will allow us to better understand the role of gutneuron-metabolic axis, to regulate energy balance in obesity and diabetes pathogenesis and novel therapeutics avenues by also tailoring probiotic interventions, as also some of us have recently envisaged [9].

To tentatively zoom down on a practical level the search of proper understanding and monitoring of intraluminal phenomena, the novel next-generation sequencing is able to analyse the complex gut polymicrobial community to allow a simultaneous identification of DNA-unique fluorescent signal. This new analysis is recently

${ }^{*}$ Corresponding author: Yadav $\mathrm{H}$, National Institute of Diabetes and Digestive and Kidney Diseases, National Institutes of Health, Bethesda, Maryland, USA Tel: 301-496-6459; E-mail: yadavhariom@gmail.com

Received June 27, 2016; Accepted June 28, 2016; Published June 30, 2016

Citation: Yadav H, Jain S, Bissi L, Marotta F (2016) Gut Microbiome Derived Metabolites to Regulate Energy Homeostasis: How Microbiome Talks to Host. Metabolomics 6: e150. doi:10.4172/2153-0769.1000e150

Copyright: ( 2016 Yadav H, et al. This is an open-access article distributed under the terms of the Creative Commons Attribution License, which permits unrestricted use, distribution, and reproduction in any medium, provided the original author and source are credited. 


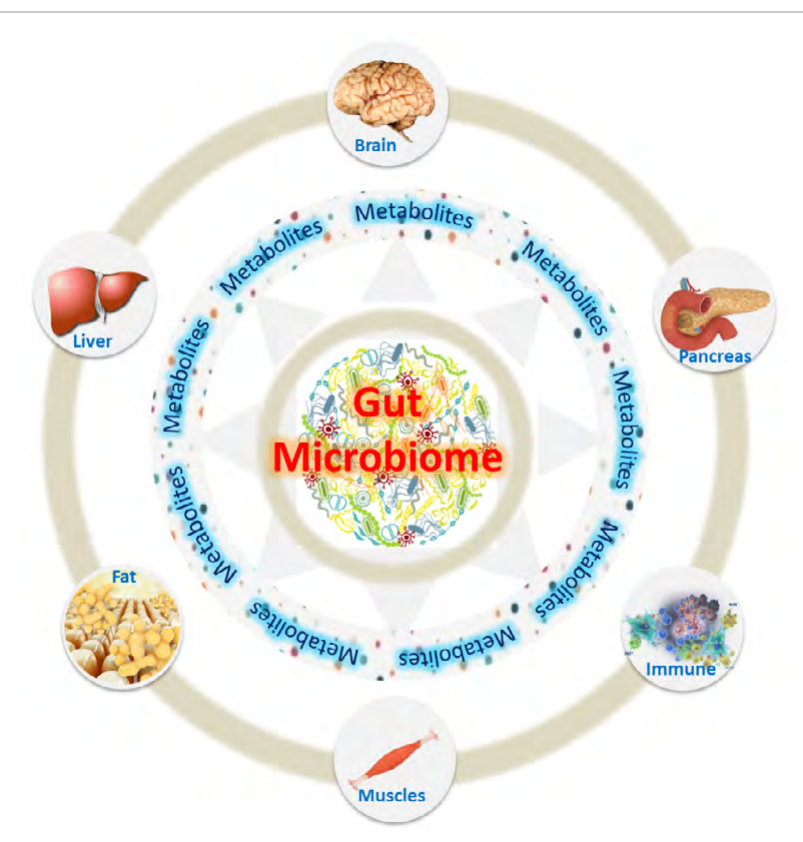

Figure 1: Microbiome derived metabolites makes direction communication with metabolic tissues of host and impact metabolic whole body function. producing a guided clinical report (Next Genomics, Prato, Italy) with very high detection accuracy. Having said that, the equally challenging conundrum remains the decoding of fundamental metabolomic pathways and their specific effectors in health and diseases.

\section{References}

1. Crow JM (2011) Microbiome: That healthy gut feeling. Nature 480: 88-89.

2. Hossain P, Kawar B, Nahas MEI (2007) Obesity and diabetes in the developing world-a growing challenge. The New England Journal of Medicine 356: 213215

3. Turnbaugh PJ, Ley RE, Mahowald MA, Magrini V, Mardis ER, et al. (2006) An obesity-associated gut microbiome with increased capacity for energy harvest. Nature 444: 1027-1031.

4. Bajzer M, Seeley RJ (2006) Physiology: obesity and gut flora. Nature 444 1009-1010.

5. Cani PD, Amar J, Iglesias MA, Poggi M, Knauf C, et al. (2007) Metabolic endotoxemia initiates obesity and insulin resistance. Diabetes 56: 1761-1772.

6. Singh V, Yeoh BS, Vijay-Kumar M (2016) Gut microbiome as a nove cardiovascular therapeutic target. Current Opinion in Pharmacology 27: 8-12.

7. Perry RJ, Peng L, Barry NA, Cline GW, Zhang D, et al. (2016) Acetate mediates a microbiome-brain-beta-cell axis to promote metabolic syndrome. Nature 534 213-217.

8. Rooks MG, Garrett WS (2016) Gut microbiota, metabolites and host immunity Nature reviews Immunology 16: 341-352.

9. Yadav H, Lee JH, Lloyd J, Walter P, Rane SG (2013) Beneficial metabolic effects of a probiotic via butyrate-induced GLP-1 hormone secretion. J Bio Chem 288: 25088-25097. 